\title{
A study of the application of fiberoptic bronchoscopy combined with liquid-based cytology test in the early diagnosis of lung cancer
}

\author{
SONGYAN HAN ${ }^{1}$, WEIHUA YANG ${ }^{1}$ and HONGWEI $\mathrm{LI}^{2}$ \\ Departments of ${ }^{1}$ Respiration Disease and ${ }^{2}$ Radiation Oncology, \\ Shanxi Provincial Cancer Hospital, Taiyuan, Shanxi 030013, P.R. China
}

Received December 11,2017; Accepted August 20, 2018

DOI: $10.3892 / 01.2018 .9372$

\begin{abstract}
Application value of fiberoptic bronchoscopy combined with liquid-based cytology test (LCT) in the early diagnosis of lung cancer was investigated. Clinical data of 901 patients who had suspicious lung space-occupying lesions and underwent bronchoscopy combined with LCT in Shanxi Provincial Cancer Hospital from June 2012 to June 2016 were retrospectively analyzed. Patients were divided into four groups to receive different fiberoptic bronchoscopies combined with LCT. Patients in Group A $(n=276)$ received bronchoscopic washing cultures (BWC), Group B $(n=204)$ received bronchoalveolar lavage $(B A L)$, Group $C(n=187)$ underwent endobronchial ultrasound-guided transbronchial needle aspiration (EBUS-TBNA) and Group D (n=234) underwent transbronchial lung biopsy (TBLB). All patients received pathological biopsy to confirm the lesions. The diagnostic results of lung cancer and the incidence rates of postoperative adverse reactions/complications were analyzed and compared among the four groups. Among 901 patients, 741 cases were pathologically diagnosed with lung cancer. In Group A, there were 224 cases diagnosed with lung cancer, of which 193 cases were successfully detected with a detection rate of $86.17 \%$ and a $\kappa$-value of 0.426 . In Group B, 171 cases were diagnosed with lung cancer, of which 149 cases were successfully detected with a detection rate of $87.13 \%$ and a $\kappa$-value of 0.430 . In Group C, 154 cases were diagnosed with lung cancer, of which 146 cases were successfully detected with a detection rate of $94.81 \%$ and a $\kappa$-value of 0.769 . In Group D, 192 cases were diagnosed with lung cancer, of which 170 cases were successfully detected with a detection rate of $88.54 \%$ and a $\kappa$-value of 0.440 . Therefore, we conclude that fiberoptic bronchoscopy
\end{abstract}

Correspondence to: Dr Songyan Han, Department of Respiration Disease, Shanxi Provincial Cancer Hospital, 3 Zhigongxin Street, Taiyuan, Shanxi 030013, P.R. China

E-mail: hnnbt0@163.com

Key words: fiberoptic bronchoscopy, liquid-based cytology, pathological biopsy, early-stage lung cancer combined with LCT technique is safe and reliable for the diagnosis of the early-stage lung cancer.

\section{Introduction}

According to reports of the World Health Organization (WHO) and other international authoritative agencies, incidence and mortality rates of lung cancer in China are increasing year by year, and China has the largest number of lung cancer patients worldwide (1-3). In spite of the efforts that have been made for the treatment of this disease, the average survival time after drug treatment is only 3-5 months (4). However, 5-year survival rates can be as high as $60-70 \%$ after early diagnosis and early operation (4). Therefore, it is of great clinical value to develop a more effective technique to improve the early diagnosis of lung cancer. Lesion tissues can be observed through a fiberoptic bronchoscope for pathological examination to definitely diagnose lung cancer. However, a fiberoptic bronchoscope is not effective for the identification of early-stage tumors due to the location or size, while cytology may compensate this deficiency. The development of cytology test techniques and the clinical application of liquid-based cytology test (LCT) technique (5-7) have significantly improved the diagnosis of chest and lung cancer, malignant pleural effusion and other non-gynecological diseases (8-11). According to the different methods used for cytology sample collection, fiberoptic bronchoscopy techniques can be divided into bronchoscopic washing cultures (BWC), bronchoalveolar lavage (BAL), endobronchial ultrasound-guided transbronchial needle aspiration (EBUS-TBNA) and transbronchial lung biopsy (TBLB) techniques (12-18). The combination of fiberoptic bronchoscopy and LCT can not only improve the diagnosis of the disease but also reduce the incidence of complications (16-18). In this study, LCT was combined with four different cytology sample-obtaining methods for the early diagnosis of lung malignant tumors, and satisfactory outcomes were reached.

\section{Patients and methods}

Study subjects. In the present retrospective study, clinical data were selected from 901 patients who received fiberoptic bronchoscopy in Shanxi Provincial Cancer Hospital (Taiyuan, 
China) from June 2012 to June 2016. The inclusion criteria were: i) patients primarily diagnosed with lung space-occupying lesions by clinical or imageological examination; ii) patients who received bronchoscopy combined with LCT; and iii) patients diagnosed by fiberoptic bronchoscopy for suspicious lesions, surgical lung biopsy or percutaneous puncture pathological examination. The 901 patients included 579 males [age range from 25 to 84 years with an average age of $57.1 \pm 10.65$ years] and 322 females [age ranged from 27 to 81 years with an average age of $55.2 \pm 11.35$ years]. All patients underwent bronchoscopy under awakened state to obtain cytology samples. All patients signed the informed consent.

Diagnostic methods. Patients with suspicious lung malignant lesions diagnosed by imageological examination were selected. According to the methods used to obtain cytology samples through bronchoscope, patients were divided into Groups A-D. Group A was treated with bronchoscopic washing cultures (BWC), Group B was treated with bronchoalveolar lavage (BAL), Group C was treated with endobronchial ultrasound-guided transbronchial needle aspiration (EBUS-TBNA) and Group D was treated with transbronchial lung biopsy (TBLB); in combination with LCT technique, respectively. At the same time, all patients received fiberoptic bronchoscopy, surgery and percutaneous puncture pathological biopsy for definite diagnosis. Patients' responses were closely observed during diagnosis and operation and their vital signs were monitored. The diagnostic results of all groups were compared and analyzed. Related indicators of four fiberoptic bronchoscopies combined with LCT in the diagnosis of lung cancer and the incidence rates of adverse reactions/complications were recorded and analyzed. This study was approved by the Ethics Committee of Shanxi Provincial Cancer Hospital.

Obtaining of cytology samples. In Group A, BWC was conducted in the suspicious malignant lung segments to obtain cytology samples; in Group B, after $15 \mathrm{ml}$ of saline at $37^{\circ} \mathrm{C}$ was perfused in the suspicious malignant lung segments through a bronchoscope, $6 \mathrm{ml}$ lavage fluid was recovered to obtain cytology samples; in Group C, a specially designed bending puncture needle was used to puncture the tissues of suspicious lesion to obtain tissues, and the obtained tissues were treated with pathological biopsy; at the same time, the residual tissues within the puncture needle were placed into an LCT tube to obtain cytology samples; in Group D, 2-4 pieces of lung tissues were taken through a pair of forceps from the suspicious malignant lung segments through a bronchoscope, and then placed and fixed in 10\% formalin solution. Cytology samples were obtained after centrifugation at $3,000 \times \mathrm{g}, 4^{\circ} \mathrm{C}$ for $15 \mathrm{~min}$ and precipitation.

LCT method. Exfoliated cytology samples obtained by fiberoptic bronchoscopy were placed into a centrifuge tube with trypsin-EDTA solution. After shaking, the tube was kept on bench for $30 \mathrm{~min}$. After gradient centrifugation at $400 \mathrm{x} \mathrm{g}, 4^{\circ} \mathrm{C}$ for $30 \mathrm{~min}$, the cells were collected. Automatic cell preparation technique was used for tissue section preparation, and tissue sections were covered with optical gum and cover glasses after Papanicolaou staining $(5,6)$.
Statistical methods. Sensitivity, specificity, positive and negative predictive values of LCT diagnosis were calculated according to pathological biopsy diagnoses. The conformity of the cytology diagnosis to the corresponding pathological biopsy in each group was estimated by $\kappa$ coefficient using the Statistical Product and Service Solutions 19.0 analysis software (IBM Corp, Armonk, NY, USA). $\kappa \geq 0.75$ indicated excellent conformity; $0.4 \leq \kappa<0.75$ indicated good conformity; $\kappa<0.4$ indicated poor conformity. ANOVA was used for the comparison of the rates among the four groups and Dunnett's test was the post hoc test used. $\mathrm{P}<0.05$ indicated that the difference was statistically significant.

\section{Results}

General data of study subjects. A total of 901 patients who underwent fiberoptic bronchoscopy in Shanxi Provincial Cancer Hospital from June 2012 to June 2016 were selected in the present study. Among the patients 579 were males and 322 were females, with an average age of 56.2 years. There was no significant difference in age between males and females in this study (Tables I and II).

Results of fiberoptic bronchoscopy combined with LCT and pathology. In Group A ( $\mathrm{n}=276), 214$ patients received BWC combined with LCT, and there were 224 patients with positive pathological biopsy results, in which 193 cases were successfully detected with a $\kappa$-value of 0.426 , indicating good conformity; in Group B ( $\mathrm{n}=204), 162$ patients received BAL combined with LCT, and there were 171 patients with positive pathological biopsy results, in which 149 patients were successfully detected with a $\kappa$-value of 0.430 , indicating good conformity; in Group C ( $\mathrm{n}=187), 151$ patients underwent EBUS-TBNA combined with LCT, and there were 154 patients with positive pathological biopsy results, in which 146 patients were successfully detected with a $\kappa$-value of 0.769 , indicating excellent conformity; in Group D ( $\mathrm{n}=234), 188$ patients underwent TBLB combined with LCT, and there were 192 patients with positive pathological biopsy results, in which 170 patients were successfully detected with a $\kappa$-value of 0.440 , indicating good conformity (Table III).

Comparison of the sensitivity (detection rate) of fiberoptic bronchoscopy combined with LCT among the four groups. Detection rate of Group A was $86.17 \%$ (193/224), and the specificity was 59.62\% (31/52); the detection rate of Group B was $87.13 \%$ (149/171), and the specificity was $60.61 \%(20 / 33)$; the detection rate of Group C was $94.81 \%$ (146/154), and the specificity was $84.85 \%$ (28/33); the detection rate of Group D was $88.54 \%$ (170/192), and the specificity was $57.14(24 / 42)$. Comparing the detection rate of the four groups: Group C $>$ Group D > Group B > Group A, and the detection rate of Group $C$ was significantly higher than those of Groups $\mathrm{A}, \mathrm{B}$ and $\mathrm{D}(\mathrm{P}<0.05)$; there was no statistically significant difference in the detection rate among Groups A, B and D. Comparing the specificity of the four groups: Group $\mathrm{C}>$ Group B $>$ Group A > Group D, and the specificity of Group C was significantly higher than those of Groups A, B and D $(\mathrm{P}<0.05)$; there was no statistically significant difference in specificity among Groups A, B and D (Tables IV and V). 
Table I. Demographic characteristics of the patients.

\begin{tabular}{lccccccccc}
\hline & \multicolumn{4}{c}{ Characteristics } & & Total & Average age (years) \\
\hline Age (years) & $20-30$ & $31-40$ & $41-50$ & $51-60$ & $61-70$ & $71-80$ & $81+$ & 579 & $57.1 \pm 10.65$ \\
Male & 15 & 21 & 113 & 207 & 169 & 53 & 1 & 322 & $55.2 \pm 11.35$ \\
Female & 9 & 20 & 66 & 121 & 80 & 24 & 2 & 501 & $56.2 \pm 11.02$ \\
Total & 24 & 41 & 179 & 328 & 249 & 77 & 3 & \\
\hline
\end{tabular}

A total of 901 patients receiving fiberoptic bronchoscopy were selected in this study, including 579 males and 322 females.

Table II. Comparison of baseline data among the four groups [n (\%)].

\begin{tabular}{|c|c|c|c|c|c|c|}
\hline Items & Group A $(n=276)$ & Group B $(n=204)$ & Group C $(n=187)$ & Group D $(n=234)$ & $\mathrm{t}$ & P-value \\
\hline Sex & & & & & 0.930 & 0.818 \\
\hline Male & $180(65.22)$ & $125(61.27)$ & $125(66.84)$ & $149(63.68)$ & & \\
\hline Female & $96(34.78)$ & $79(38.73)$ & $62(33.16)$ & $85(36.32)$ & & \\
\hline Age (years) & & & & & 7.676 & 0.053 \\
\hline$<58$ & $121(43.84)$ & $115(56.38)$ & $90(48.13)$ & $110(47.01)$ & & \\
\hline$\geq 58$ & $155(56.16)$ & $89(43.63)$ & 97 (51.87) & $124(52.99)$ & & \\
\hline Smoking & & & & & 0.295 & 0.961 \\
\hline Yes & $98(35.51)$ & $68(33.33)$ & $63(33.69)$ & $80(34.19)$ & & \\
\hline No & $178(64.49)$ & $136(66.67)$ & $124(66.31)$ & $154(65.81)$ & & \\
\hline
\end{tabular}

Comparison of the incidence rates of complications/adverse reactions among four groups. In Group A ( $\mathrm{n}=276)$, hemoptysis occurred in 1 patient, and the incidence of complications/adverse reactions was $0.36 \%$; in Group B $(n=204)$, tracheal injury occurred in 1 patient, and the incidence of complications/adverse reactions was $0.49 \%$; in Group C $(n=187)$, hemorrhage occurred in 1 patient, and the incidence rate of complications/adverse reactions was $0.53 \%$; in Group D $(n=234)$, pneumothorax occurred in 2 patients with a pneumothorax quantity of $\sim 9.5 \%$, and the incidence rate of complications/adverse reactions was $0.85 \%$; the differences in the incidences of complications/adverse reactions among the four groups were not statistically significant $(\mathrm{p}>0.05)$ (Table VI).

\section{Discussion}

With the unacceptably high morbidity and mortality rates, lung cancer is one of the most common malignant tumors that endanger the lives and health of humans around the world. Cytology detection through fiberoptic bronchoscopy using cytology samples has become one of the most commonly used methods for the diagnosis of lung cancer. With the application of LCT technique, the accuracy of the diagnosis of lung cancer by cytology has been significantly improved. LCT also plays an important role in early diagnosis of lung cancer. In this study, clinical data from 901 patients with suspicious lung cancer who underwent fiberoptic bronchoscopy combined with LCT and pathological biopsy within the last four years were retrospectively analyzed and summarized. With histopathological diagnosis as gold standard, as well as the incidence rate of complications/adverse
Table III. Results of fiberoptic bronchoscopy combined with LCT and pathology as well as conformity $(n, \kappa)$.

Pathological tissue

LCT Positive Negative Total $\kappa$-value

\begin{tabular}{lrrrr}
\hline Group A (BWC) & & & & \\
Positive & 193 & 21 & 214 & 0.426 \\
Negative & 31 & 31 & 62 & \\
Subtotal & 224 & 52 & 276 & \\
Group B (BAL) & & & & \\
Positive & 149 & 13 & 162 & 0.430 \\
Negative & 22 & 20 & 42 & \\
Subtotal & 171 & 33 & 204 & \\
Group C (EBUS-TBNA) & & & & \\
Positive & 146 & 5 & 151 & 0.769 \\
Negative & 8 & 28 & 36 & \\
Subtotal & 154 & 33 & 187 & \\
Group D (TBLB) & & & & \\
Positive & 170 & 18 & 188 & 0.440 \\
Negative & 22 & 24 & 46 & \\
Subtotal & 192 & 42 & 234 & \\
Total & 741 & 160 & 901 &
\end{tabular}

LCT, liquid-based cytology test; BWC, bronchoscopic washing cultures; BAL, bronchoalveolar lavage; EBUS-TBNA, endobronchial ultrasound-guided transbronchial needle aspiration; TBLB, transbronchial lung biopsy. 
Table IV. Comparison of the detection rate of fiberoptic bronchoscopy combined with LCT among the four groups (\%).

\begin{tabular}{|c|c|c|c|c|c|c|}
\hline $\begin{array}{l}\text { Fiberoptic } \\
\text { bronchoscopy } \\
\text { combined } \\
\text { with LCT }\end{array}$ & Sensitivity $^{\mathrm{a}}$ & Specificity & $\begin{array}{l}\text { Positive } \\
\text { predictive } \\
\text { value }\end{array}$ & $\begin{array}{c}\text { Negative } \\
\text { predictive } \\
\text { value }\end{array}$ & $\begin{array}{c}\text { False } \\
\text { positive } \\
\text { rate }\end{array}$ & $\begin{array}{c}\text { False } \\
\text { negative } \\
\text { rate }\end{array}$ \\
\hline Group A (BWC) & $86.17 \%(193 / 224)$ & $59.62 \%(31 / 52)$ & $90.19 \%(193 / 214)$ & $50.00 \%(31 / 62)$ & $40.38 \%(21 / 52)$ & $13.84 \%(31 / 224)$ \\
\hline Group B (BAL) & $87.13 \%(149 / 171)$ & $60.61 \%(20 / 33)$ & $91.98 \%(149 / 162)$ & $47.62 \%(20 / 42)$ & $39.39 \%(13 / 33)$ & $12.87 \%(22 / 171)$ \\
\hline $\begin{array}{l}\text { Group C } \\
\text { (EBUS-TBNA) }\end{array}$ & $94.81 \%(146 / 154)$ & $84.85 \%(28 / 33)$ & $96.69 \%(146 / 151)$ & $77.78 \%(28 / 36)$ & $15.15 \%(5 / 33)$ & $5.19 \%(8 / 154)$ \\
\hline Group D (TBLB) & $88.54 \%(170 / 192)$ & $57.14(24 / 42)$ & $90.43 \%(170 / 188)$ & $52.17 \%(24 / 46)$ & $42.86 \%(18 / 42)$ & $11.46 \%(22 / 192)$ \\
\hline$\chi^{2}$ & 7.642 & 7.707 & 6.118 & 8.094 & 6.634 & 7.642 \\
\hline P-value & 0.054 & 0.052 & 0.106 & 0.044 & 0.085 & 0.054 \\
\hline
\end{tabular}

${ }^{a}$ Detected sensitivity/detection rate $=$ the number of cases successfully diagnosed with lung cancer through pathology by fiberoptic bronchoscopy combined with LCT within the group/the number of cases pathologically diagnosed with lung cancer within the group x100\%. LCT, liquid-based cytology test; BWC, bronchoscopic washing cultures; BAL, bronchoalveolar lavage; EBUS-TBNA, endobronchial ultrasound-guided transbronchial needle aspiration; TBLB, transbronchial lung biopsy.

Table V. Comparison of false negative rate among the four groups.

\begin{tabular}{lcc}
\hline Control group & $\chi^{2}$ & P-value \\
\hline A (BWC) & & \\
B (BAL) & 0.079 & 0.778 \\
C (EBUS-TBNA) & 7.370 & 0.007 \\
D (TBLB) & 0.527 & 0.468 \\
B (BAL) & & \\
C (EBUS-TBNA) & 5.690 & 0.017 \\
D (TBLB) & 0.168 & 0.682 \\
C (EBUS-TBNA) & & 0.040 \\
D (TBLB) & 4.234 & \\
\hline
\end{tabular}

BWC, bronchoscopic washing cultures; BAL, bronchoalveolar lavage; EBUS-TBNA, endobronchial ultrasound-guided transbronchial needle aspiration; TBLB, transbronchial lung biopsy.

reactions, application values of bronchoscopy combined with LCT technique in the early diagnosis of lung cancer were evaluated.

In the present study, the sensitivity (detection rate), specificity and $\kappa$-value gained from the statistical data analysis of Group A/B/C/D were basically similar to those reported in previous studies $(5,17,19)$. Sun et al $(20)$ also revealed that EBUS-TBNA is the most advanced technique in the collection of cytology samples through microscopic examination. With EBUS-TBNA, tumor samples can be obtained that are not accessible by conventional techniques. Based on statistical analyses, it is not difficult to find that BWC/BAL/EBUS-TBNA/TBLB combined with LCT can reduce omission diagnose rate and misdiagnose rate of lung cancer, so they can be used as effective detection techniques for the early diagnosis of lung cancer. Fiberoptic bronchoscopy combined with LCT, as an important technique for early diagnosis of lung tumors, has the following advantages: i) simple operation, small trauma and high positive diagnosis rate; ii) few pneumothorax, hemorrhage, lung infection and other complications/adverse reactions; iii) avoidance of radiation from X-ray and computed tomography (CT)-guided percutaneous puncture biopsy/transmural biopsy to medical personnel and patients; and iv) less pain suffered by patients during operation, thus repeated examinations can be performed. In addition, the use of LCT fundamentally changes the traditional smear production methods and compensates their deficiencies. As a result, advantages of fiberoptic bronchoscopy combined with LCT are: i) access to cytology samples to the utmost possibility with less cell destruction; ii) maintenance of cytology samples to the utmost possibility during smear process; and iii) single-layer uniformly distributed smears and concentrated cells with clear structures, which are conducive to better radiograph reading experience.

Regarding the false positive cases in each group, there were 57 cases of false positive cases of liquid-based cytology in the four groups. Histopathological examinations diagnosed those cases as tuberculosis, hamartoma, and lung abscesses and other inflammatory infections. Cytological diagnosis is mainly based on heteromorphism of cells and nucleus morphology. Abnormal cell shape is often used as an important basis for diagnosing malignant transformation. Changes in proliferation, metaplasia, and degeneration of lung cells that occur during inflammatory infections may lead to varying degrees of morphological changes, which in turn affect the diagnosis and lead to the false positive diagnosis. In addition, the appearance of tumor without obvious malignant features and well-differentiated tumor has similarities with hyperplastic cells, which is another cause of false positive diagnosis. Fernández-Bussy et al (21) also reported that the application of pulmonary lesions cytology and biopsy may misidentify malignant cells, resulting in false positive and false negative diagnosis. Therefore, repeated examinations and combination of imaging techniques are needed to improve the diagnosis. 
Table VI. Comparison of the incidence rates of complications/adverse reactions among the four groups.

\begin{tabular}{|c|c|c|c|c|c|c|c|c|}
\hline Group & $\begin{array}{c}\text { Hemorrhage/ } \\
\text { hemoptysis }\end{array}$ & Hemothorax & Pneumothorax & $\begin{array}{l}\text { Lung } \\
\text { infection }\end{array}$ & $\begin{array}{c}\text { Tracheal } \\
\text { injury/spasm }\end{array}$ & Hyoxemia & $\begin{array}{l}\text { Arrhythmia/ } \\
\text { heart failure }\end{array}$ & $\begin{array}{l}\text { Total } \\
\mathrm{n}(\%)\end{array}$ \\
\hline Group A $(n=276)$ & 1 & 0 & 0 & 0 & 0 & 0 & 0 & $1(0.36)$ \\
\hline Group B $(n=204)$ & 0 & 0 & 0 & 0 & 1 & 0 & 0 & $1(0.49)$ \\
\hline Group C $(n=187)$ & 1 & 0 & 0 & 0 & 0 & 0 & 0 & $1(0.53)$ \\
\hline Group D $(n=234)$ & 0 & 0 & 2 & 0 & 0 & 0 & 0 & $2(0.85)$ \\
\hline$\chi^{2}$ & 2.046 & & 5.741 & & 3.618 & & & 0.583 \\
\hline P-value & 0.563 & & 0.126 & & 0.306 & & & 0.900 \\
\hline
\end{tabular}

Besides, normal tissues may be collected by bronchoscopy under direct vision, which in turn leads to false negative cases. However, the application of EBUS-TBNA technology in Group $\mathrm{C}$ is a good way to solve this defect because it can be used to obtain cytological samples under visible conditions, leading to the lowest false negative rate among the four groups.

It is known that collection of tissue samples by fiberoptic bronchoscopy may lead to various complications or adverse reactions (21). However, in this study, the incidence of complications/adverse reactions in Group A/B/C/D was low, and there were no statistically significant differences in incidences of complications/adverse reactions among the four groups, which is consistent with the findings reported by Chan et al (22) and Asano et al (23). Although pneumothorax occurred in 2 patients in Group D $(n=234)$, pneumothorax quantity was only $9.5 \%$. Patients combined with pneumothorax were able to conduct self-absorption without treatment. It has been reported (24) that the incidence rate of pneumothorax was $10-40 \%$ and that of hemoptysis was $20-33 \%$ after CT-guided percutaneous puncture biopsy. However, the incidence of pneumothorax and hemoptysis of patients in this study were significantly lower than those of patients who received percutaneous biopsy, possibly due to the small trauma and simple operation. Incidence of lung cancer is significantly increased with aging (25). Silvestri et al (26) showed that the safety of performing bronchoscopy under sodium-fluorophosphate anesthesia for aged population is comparable to that of young individuals. Therefore, the combination of BWC/BAL/EBUS-TBNA/TBLB and LCT is also safe for aged population.

Since histopathological diagnosis is the gold standard for the diagnosis of lung cancer and lung cancer classification, cytology diagnosis cannot be used to guide the treatment. All patients included in this study were pathologically diagnosed by bronchoscopic biopsy, while patients only diagnosed by bronchoscopy combined with liquid-based cytology were not included, which is a shortcoming of this study. However, tissue contamination and missed labeling may lead to misdiagnosis of histopathological examination. Therefore, repeated diagnosis or combination with clinical manifestations and imaging examination is also necessary to achieve accurate diagnosis.

In summary, the four methods (BWC/BAL/EBUS TBNA/TBLB) combined with LCT are safe and reliable techniques for the early diagnosis of lung cancer. However, the combination of EBUS-TBNA and LCT is the most promising one. LCT technique cannot completely replace conventional histopathological biopsy, but LCT is indispensable for the diagnosis of early-stage lung cancer due to the difficulties in tissue collection through conventional methods. Therefore, combining the two methods can improve the early diagnosis of lung cancer.

\section{Acknowledgements}

Not applicable.

\section{Funding}

No funding was received.

\section{Availability of data and materials}

The datasets used and/or analyzed during the present study are available from the corresponding author on reasonable request.

\section{Authors' contributions}

SH conceived, designed the study and drafted the manuscript. SH, WY and HL collected, analyzed, and interpreted the patient data, and revised the manuscript critically for important intellectual content. All authors read and approved the manuscript and agree to be accountable for all aspects of the research in ensuring that the accuracy or integrity of any part of the study are appropriately investigated and resolved.

\section{Ethics approval and consent to participate}

The study was approved by the Ethics Committee of Shanxi Provincial Cancer Hospital (Taiyuan, China). Signed informed consents were obtained from the patients or the guardians.

\section{Patient consent for publication}

Not applicable.

\section{Competing interests}

The authors declare that they have no competing interests. 


\section{References}

1. She J, Yang P, Hong Q and Bai C: Lung cancer in China: Challenges and interventions. Chest 143: 1117-1126, 2013.

2. Jemal A, Bray F, Center MM, Ferlay J, Ward E and Forman D: Global cancer statistics. CA Cancer J Clin 61: 69-90, 2011.

3. Chen WQ, Zhang SW, Zou XN and Zhao P: An analysis of lung cancer mortality in China, 2004-2005. Zhonghua Yu Fang Yi Xue Za Zhi 44: 378-382, 2010 (In Chinese).

4. Siegel R, Naishadham D and Jemal A: Cancer statistics, 2012. CA Cancer J Clin 62: 10-29, 2012.

5. Chen H, Shu HM, Chang ZL, Wang ZF, Yao HH, Zhu HM, Lu TM, Ma QY and Yang BL: Efficacy of Pap test in combination with ThinPrep cytological test in screening for cervical cancer. Asian Pac J Cancer Prev 13: 1651-1655, 2012.

6. Tripathy K, Misra A and Ghosh JK: Efficacy of liquid-based cytology versus conventional smears in FNA samples. J Cytol 32: $17-20,2015$.

7. Rouprêt M: Reply to Yan Shibing and Wei Qiang's letter to the Editor re: Morgan Rouprêt, Marko Babjuk, Eva Compérat, et al. European Association of Urology Guidelines on Upper Urinary Tract Urothelial Cell Carcinoma: 2015 Update. Eur Urol 2015;68:868-79. Eur Urol 69: e51-e52, 2016.

8. Fadda G and Rossi ED: Liquid-based cytology in fine-needle aspiration biopsies of the thyroid gland. Acta Cytol 55: 389-400, 2011.

9. Rarick JM, Wasman J and Michael CW: The utility of liquid-based cytology in salivary gland fine-needle aspirates: Experience of an academic institution. Acta Cytol 58: 552-562, 2014.

10. Liu C, Wen Z, Li Y and Peng L: Application of ThinPrep bronchial brushing cytology in the early diagnosis of lung cancer: A retrospective study. PLoS One 9: e90163, 2014.

11. Travis WD, Brambilla E, Noguchi M, Nicholson AG, Geisinger K, Yatabe Y, Ishikawa Y, Wistuba I, Flieder DB Franklin W, et al: Diagnosis of lung cancer in small biopsies and cytology: Implications of the 2011 International Association for the Study of Lung Cancer/American Thoracic Society/European Respiratory Society classification. Arch Pathol Lab Med 137: 668-684, 2013.

12. Idowu MO and Powers CN: Lung cancer cytology: Potential pitfalls and mimics - a review. Int J Clin Exp Pathol 3: 367-385, 2010.

13. Wright PK, Thiryayi SA and Rana DN: Fine needle aspiration cytology diagnosis of extramedullary haematopoiesis presenting as a pre-sacral mass: A pitfall avoided. Cytopathology 23: 133-134, 2012.

14. Tabatabai ZL, Auger M, Kurtycz DF, Laucirica R, Souers RJ, Nayar R, Khalbuss WE, Moriarty AT and Fraig M: Do liquid-based preparations of pulmonary bronchial brushing specimens perform differently from classically prepared cases for the diagnosis of malignancies? Observations from the College of American Pathologists interlaboratory comparison program in nongynecologic cytology. Arch Pathol Lab Med 139: 178-183, 2015.

15. Osińska I and Domagała-Kulawik J: Bronchoalveolar lavage in lung cancer - diagnostic value and assessment of the anti-cancer immune response. Postepy Hig Med Dosw 67: 1119-1127, 2013.
16. Fukuhara N, Tachihara M, Tanino Y, Saito J, Sato S, Nikaido T, Misa K, Fukuhara A, Wang X, Ishida T, et al: Cholesteryl palmitate crystals in bronchoalveolar lavage fluid smears as a possible prognostic biomarker for chronic interstitial pneumonia: A preliminary study. Respir Investig 54: 109-115, 2016.

17. Morimoto A, Ito A, Hashimoto K, Nakano A, Nagasaka T and Yokoi T: New diagnostic technique for rapid fluorescence immunocytochemical staining of adenocarcinoma and mesothelial cells using liquid-based cytology. Acta Cytol 58: 461-468, 2014.

18. Minami D, Takigawa N, Morichika D, Kubo T, Ohashi K, Sato A Hotta K, Tabata M, Tanimoto M and Kiura K: Endobronchial ultrasound-guided transbronchial biopsy with or without a guide sheath for diagnosis of lung cancer. Respir Investig 53: 93-97, 2015.

19. Sing A, Freudenberg N, Kortsik C, Wertzel H, Klosa B and Hasse J: Comparison of the sensitivity of sputum and brush cytology in the diagnosis of lung carcinomas. Acta Cytol 41: 399-408, 1997.

20. Sun H, Sun X, Zhai X, Guo J, Liu Y, Ying J and Wang Z: Body mass index and exon 19 mutation as factors predicting the therapeutic efficacy of gefitinib in patients with epidermal growth factor receptor mutation-positive non-small cell lung cancer. Thorac Cancer 7: 61-65, 2016.

21. Fernández-Bussy S, Labarca G, Zagolin M, Oyonarte M, Isamit D, Jalilie A, Guerra C and Chernilo S: Immediate complications following flexible bronchoscopy: Retrospective analysis of 1079 procedures (In Spanish). Rev Med Chil 142: 299-304, 2014.

22. Chan JW, Chu SY, Lam CH, O WH, Cheung OY, Kwan TL, Leung AK and Law WL: Pulmonary artery sarcoma diagnosed by endobronchial ultrasound-guided transbronchial needle aspiration. Hong Kong Med J 20: 152-155, 2014.

23. Asano F, Aoe M, Ohsaki Y, Okada Y, Sasada S, Sato S, Suzuki E, Semba H, Fukuoka K, Fujino S, et al: Complications associated with endobronchial ultrasound-guided transbronchial needle aspiration: A nationwide survey by the Japan Society for Respiratory Endoscopy. Respir Res 14: 50, 2013.

24. Uruga $H$, Takaya $H$, Hanada S, Beika Y, Miyamoto A, Morokawa N, Kurosaki A, Fujii T and Kishi K: Diagnostic efficacy of CT-guided transthoracic needle biopsy and fine needle aspiration in cases of pulmonary infectious disease. Jpn J Radiol 30: 589-593, 2012.

25. Wood DE: National Comprehensive Cancer Network (NCCN) Clinical practice guidelines for lung cancer screening. Thorac Surg Clin 25: 185-197, 2015.

26. Silvestri GA, Vincent BD and Wahidi MM: Fospropofol disodium for sedation in elderly patients undergoing flexible bronchoscopy. J Bronchology Interv Pulmonol 18: 15-22, 2011. 